\title{
3D CT airway evaluation-guided intraluminal placement of endobronchial blocker in pediatric patients: a randomized controlled study
}

\author{
Yingyi Xu ${ }^{1}$, Le $\mathrm{Li}^{2}$, Jianning Hou ${ }^{3}$, Na Zhang ${ }^{1}$, Minting Zeng ${ }^{1}$, Qianqi Qiu ${ }^{1}$, Yufeng Liang ${ }^{4}$, Wei Wei ${ }^{1}$, \\ Yonghong $\operatorname{Tan}^{1}$ \\ ${ }^{1}$ Department of Anesthesiology, Guangzhou Women and Children's Medical Center, Guangzhou Medical University, Guangzhou, China; \\ ${ }^{2}$ Department of Pediatric Surgery, Guangzhou Women and Children's Medical Center, Guangzhou Medical University, Guangzhou, China; \\ ${ }^{3}$ Department of Radiology, Guangzhou Women and Children's Medical Center, Guangzhou Medical University, Guangzhou, China; ${ }^{4}$ Pediatric \\ Intensive Care Unit, Guangzhou Women and Children's Medical Center, Guangzhou Medical University, Guangzhou, China \\ Contributions: (I) Conception and design: Y Xu, Y Tan; (II) Administrative support: L Li, N Zhang; (III) Provision of study materials or patients: \\ Y Xu, L Li, J Hou, Y Tan; (IV) Collection and assembly of data: Y Xu, Y Tan, Y Liang; (V) Data analysis and interpretation: M Zeng, Q Qiu; (VI) \\ Manuscript writing: All authors; (VII) Final approval of manuscript: All authors. \\ Correspondence to: Yonghong Tan. Department of Anesthesiology, Guangzhou Women and Children’s Medical Center, 9 Jinsui Road, Guangzhou \\ 510623, China. Email: yonghongtandoctor@yeah.net.
}

Background: The aim of the present study was to propose a new approach for 3D computed tomography (CT) airway evaluation-guided endobronchial blocker placement in pediatric patients, and to determine its efficiency in clinical application.

Methods: A total of 127 pediatric patients aged 0.5-3 years who were scheduled for elective thoracic surgery using one-lung ventilation (OLV) were randomized into the bronchoscopy (BRO) group and the CT group. The degree of lung collapse, postoperative airway mucosal injury, pulmonary infection within $72 \mathrm{~h}$ after surgery, and hoarseness after tracheal extubation; duration of postoperative mechanical ventilation, intensive care unit (ICU) stay and hospitalization; success rate of first blocker positioning; and required time and repositioning for successful blocker placement were compared between the 2 groups.

Results: The degree of lung collapse, postoperative airway mucosal injury, pulmonary infection within $72 \mathrm{~h}$ after surgery, and hoarseness after tracheal extubation; duration of postoperative mechanical ventilation, ICU stay and hospitalization; success rate of first blocker positioning; and required time and repositioning for successful blocker placement were similar between the 2 groups (all $\mathrm{P}>0.05$ ).

Conclusions: For pediatric patients undergoing surgery with OLV, preoperative 3D CT airway evaluation could be used to guide endobronchial blocker placement, with a blocking efficiency similar to that of BROguided blocker placement.

Keywords: Endobronchial blocker; pediatric patients; 3D computed tomography (3D CT); one-lung ventilation $(\mathrm{OLV})$; bronchoscopy (BRO)

Submitted Dec 23, 2020. Accepted for publication Mar 05, 2021.

doi: $10.21037 / \mathrm{tp}-21-33$

View this article at: http://dx.doi.org/10.21037/tp-21-33

\section{Introduction}

During anesthesia for thoracic surgery, one-lung ventilation (OLV) is important because lung isolation is necessary for most thoracic surgeries to ensure clear surgical exposure
(1-3). Bronchial blocking is the most commonly used OLV technique for pediatric patients $(4,5)$. Bronchoscopy (BRO) is commonly used to guide endobronchial blocker placement in pediatric patients. Blocking techniques 


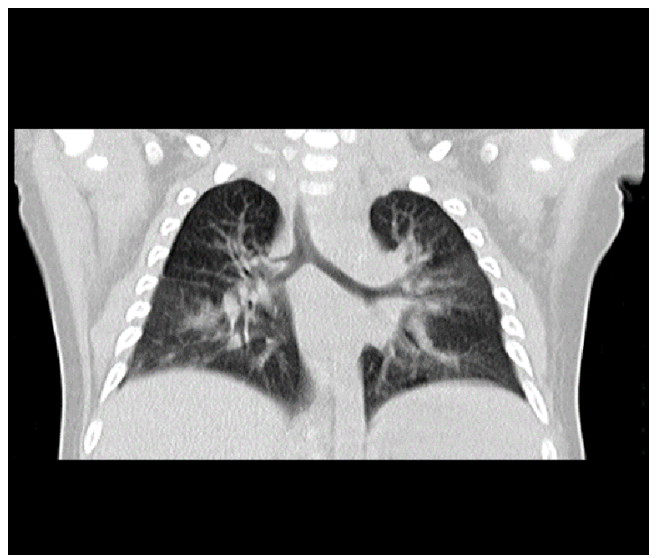

Figure 1 Opening of the right upper lung lobe is parallel to or higher than the carina.

include intraluminal and extraluminal blocking $(6,7)$; however, intraluminal blocking causes less injury with certain blocking efficiency, but only the tracheal tube with an internal diameter $>4.5 \mathrm{~mm}$ could allow the simultaneous insertion of the bronchoscope and the finest bronchial blocker (8). BRO-guided intraluminal blocking cannot be applied in pediatric patients who are too young or have a narrow airway that cannot allow the insertion of a tracheal tube with an internal diameter $>4.5 \mathrm{~mm}$. In addition, for institutions without fiberoptic BRO, how to properly place the endobronchial blocker in pediatric patients remains challenging.

Chest computed tomography (CT) images could be used to accurately predict the optimal insertion depth of the double-lumen tracheal tube and guide extraluminal uniblocker placement in the left bronchus in adult patients $(9,10)$. However, the distance from the carina to the opening of the main bronchus is different between children and adults. Whether chest CT images could be used to guide endobronchial blocker placement in pediatric patients remains to be determined. We proposed a novel method of 3D CT evaluation to measure the airway and guide endobronchial blocker placement in pediatric patients. In the present randomized prospective study, we compared the efficiency of endobronchial blocker placement guided by $\mathrm{BRO}$ and 3D CT reconstruction and determined the feasibility of applying preoperative helical 3D CT airway reconstruction to guide endobronchial blocker placement in pediatric patients. We present the following article in accordance with the STROBE reporting checklist (available at http://dx.doi.org/10.21037/tp-21-33).

\section{Methods}

\section{Patient enrollment and randomization}

The present study was approved by the institutional ethical committee of Guangzhou Women and Children's Medical Center (No. 2014051229, approval date: June 3, 2014). The trial was registered prior to patient enrollment at China Clinical Trial Registry (http://www.chictr.org.cn/ showproj.aspx?proj=4344, principal investigator: Yingyi $\mathrm{Xu}$, registration number: ChiCTR-TRC-14005232, date of registration: August 12, 2014). All procedures involving human participants were done in accordance with the Helsinki Declaration (as revised in 2013), and written informed consent was obtained from all patient's parents/guardians enrolled in the study. Images relating to participants in the manuscript were obtained with written informed consent from the parents/guardians. Pediatric patients scheduled for elective thoracic surgery between September 2014 and June 2016 at Guangzhou Women and Children's Medical Center were selected. The enrollment criteria were as follows: (I) American society of Aneshesiologists (ASA) stage I-III; and (II) aged 0.5-3 years. The exclusive criteria were as follows: (I) airway compression; (II) laryngeal edema or acute airway inflammation; (III) opening of the right upper lung lobe was parallel to or higher than the carina (Figure 1); (IV) patients suspected to have difficulty during laryngoscopy and in airway management. The enrolled patients were randomized into the BRO group and the CT group using the closed envelope technique. Once the grouping was completed, if children could not be introduced endotracheal tube of $4.5 \mathrm{~mm}$, they would withdraw from the study. Random numbers were generated using SAS software (version 9.2; SAS Institute Inc., Cary, NC, USA) at a ratio of 1:1. These numbers were then sealed in envelopes and kept by an independent study coordinator, perioperative care, and postoperative follow-up of the patients. The endobronchial blocker placement was guided by BRO in the BRO group and by 3D CT airway evaluation in the CT group. All cases of anesthesia were performed by a pediatric anesthetist with 6 years' experience in thoracic anesthesia. During the study period, patients were consecutively recruited and randomly divided into the control or intervention groups accordingly. The study was single blinded. Anesthesiologists who administered anesthesia were not involved in the patients' follow up and data collection. Patients, health-care providers, and investigators who were in charge of followup and data collection were blinded to the study protocol. 


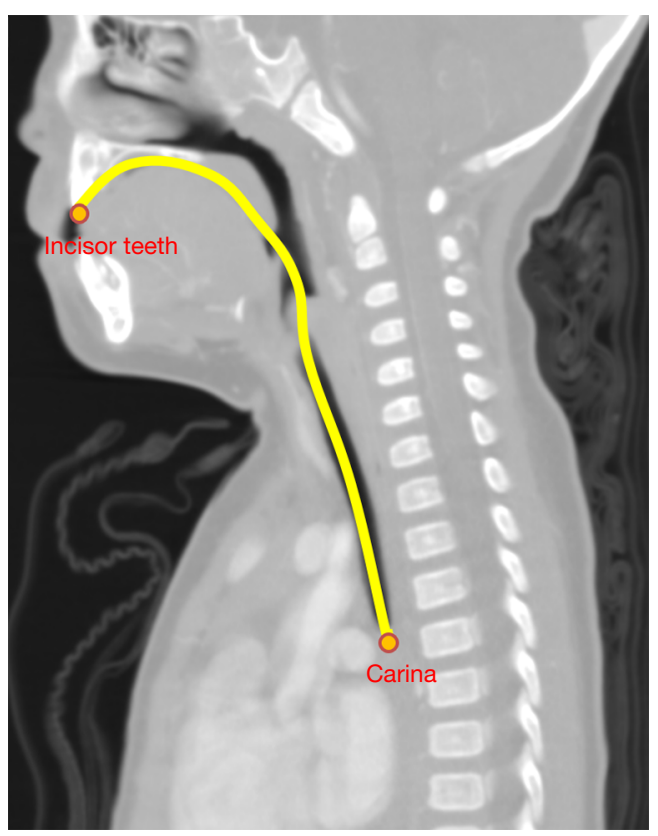

Figure 2 Measure of the distance from the incisor teeth to the carina.

\section{CT measurement}

After sedation, all pediatric patients under sedation preoperative cervical and chest CT scanning (Aquilion 64, Toshiba, Dalian, Liaoning, China) in the supine position. A lung window was applied to reconstruct coronal and sagittal image with $3-\mathrm{mm}$ slices and $3-\mathrm{mm}$ slice gaps and $512 \times 512$ image resolutions. The Frankfurt horizontal plane was confirmed with bilateral auriculares and the right infraorbital margin; the midsagittal plane was confirmed with the middle of the sella turcica, the nasion, and the posterior edge of the foramen magnum. The distance from the incisor teeth to the tracheal carina was measured using the sagittal image when the incisor teeth, the glottis and the whole airway were able to be exposed clearly at the same time. In the case of incorrect positioning or airway compression, multiplanar reconstruction or curve planar reconstruction was applied (Figure 2).

\section{Anesthesia}

All pediatric patients received an intravenous injection of $0.01 \mathrm{mg} / \mathrm{kg}$ phencyclidine hydrochloride before surgery and oxygen inhalation after entering the operation room. They received a micro-pump infusion $(8-10 \mathrm{~mL} / \mathrm{kg} / \mathrm{h})$ of sodium acetate Ringer's injection, and their Blood Pressure (BP),
Heart Rate (HR), electrocardiogram (ECG), and Saturation of Pulse Oxygen $\left(\mathrm{SpO}_{2}\right)$ were monitored. Propofol $(2 \mathrm{mg} / \mathrm{kg})$, sufentanil $(0.3 \mu \mathrm{g} / \mathrm{kg})$, and cisatracurium $(0.2 \mathrm{mg} / \mathrm{kg})$ were injected intravenously to induce general anesthesia. A tracheal tube without side holes (WeiLi Medical, Guangzhou, China) was then intubated using laryngoscopy. If the patient was aged 1-3 year, the model of endotracheal tubes was selected according to the classic formula (based on predicted age formula). If the patient was aged $0.5-1$ year, ETT of $4.5 \mathrm{~mm}$ was tried to insert. After intubation, the partial pressure of carbon dioxide in endexpiratory gas, as well as invasive arterial blood pressure and central venous pressure, were monitored, and suctioning was performed. Inhalation of $1-3 \%$ sevoflurane was used for anesthesia maintenance with a tidal volume of $6-8 \mathrm{~mL} / \mathrm{kg}$. The concentration of sevoflurane was adjusted according to hemodynamic changes and data of anesthesia monitoring. Cisatracurium and sufentanil were supplemented as necessary. All patients were transferred to the intensive care unit (ICU) after surgery.

\section{Endobronchial blocker placement}

In the CT group, 3D CT evaluation images were used to measure the length of the main bronchus (the length from the incisor teeth to the carina) before endobronchial blocker placement. Prior to endotracheal intubation, the insertion depth was preset as the CT-measured length of the main bronchus minus $2 \mathrm{~cm}$ and was marked as marker 1 on the tracheal tube (Figure 3A). The endobronchial blocker was inserted through the tracheal tube until point A of the balloon reached the tube tip. The positions on the blocker which paralleled the screw cap (marker 2) and the screw cap plus $2 \mathrm{~cm}$ (marker 3 ) were marked, and the endobronchial blocker was then extubated after the cap was screwed on (Figure 3B). The tracheal tube was inserted into marker 1 under direct-vision laryngoscopy. The endobronchial blocker was reinserted through the tracheal tube. The connectors of the endobronchial blocker and the tracheal tube were fixed when the screw cap paralleled marker 2 . The endobronchial blocker was further inserted until the screw cap paralleled marker 3 with resistance disappeared, and the balloon was inflated with $1.5-2.5 \mathrm{~mL}$ of air (Figure 3C). Both lungs were auscultated to ensure that respiratory sounds disappeared in the lung of the blocking side. If proper blocking was not achieved after 3 consecutive repositioning trials, BRO-guided placement was applied, and the patient was excluded from the study. Proper blocker 


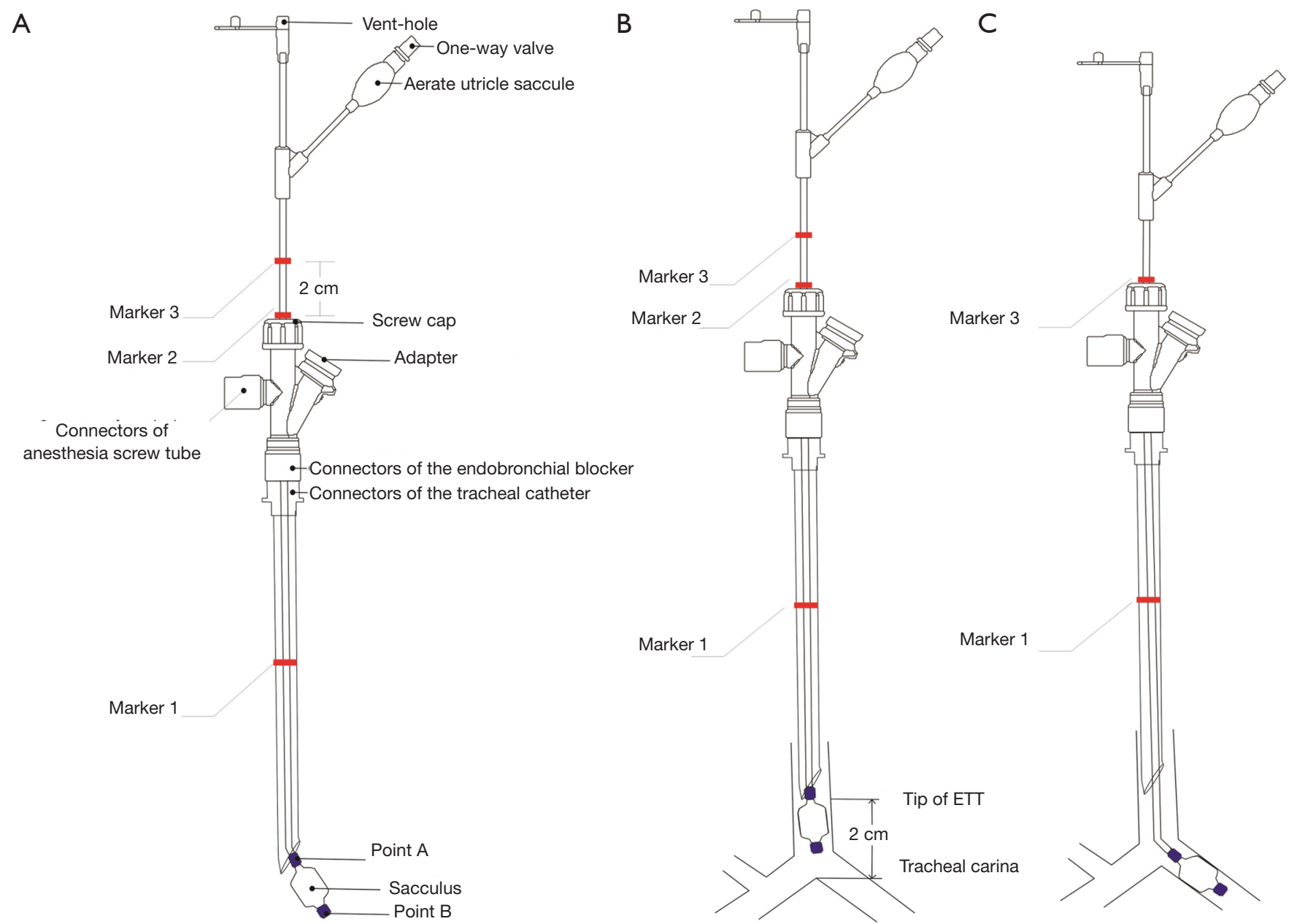

Figure 3 Process to locate the bronchial blocker (computed tomography group). (A) Depth of intubation was preset as the CT-measured length of the main bronchus minus $2 \mathrm{~cm}$; (B) the sketch Map of position of the bronchial blocker and the bronchus; (C) the endobronchial blocker was further inserted.

placement was confirmed by auscultation after the patient was transferred to lateral position.

In the BRO group, the insertion depth of the tracheal tube was calculated using the formula of tracheal intubation depth in children (11). After the 5-F WeiLi endobronchial blocker (WeiLi Medical, China) was placed into the tracheal tube, an electrobronchoscope (A20-2.8; Maidehao, Zhuhai, Guangdong, China), with a diameter of $2.8 \mathrm{~mm}$, was inserted to help locate the endobronchial blocker until the point A of the endobronchial blocker reached the opening of the main bronchus at the blocking side (Figure 4). Proper blocker placement was confirmed under BRO after the patients were moved from the horizontal position to the lateral position.

\section{Observational parameters}

The observational parameters were as follows: (I) the required time for successful blocker placement (measured when the endobronchial blocker was inserted through the vocal cord until it was placed at the proper position); (II) the number of repositioning trials for successful blocker placement (each extubation of the endobronchial blocker from the tracheal tube was counted as 1 repositioning trial); (III) the success rate of the first blocker positioning; (IV) the degree of lung collapse, ranked by the surgeon as excellent (complete lung collapse at the blocking side), fair (lung collapse at the blocking side with a little amount of residual air that would not affect surgical exposure), 


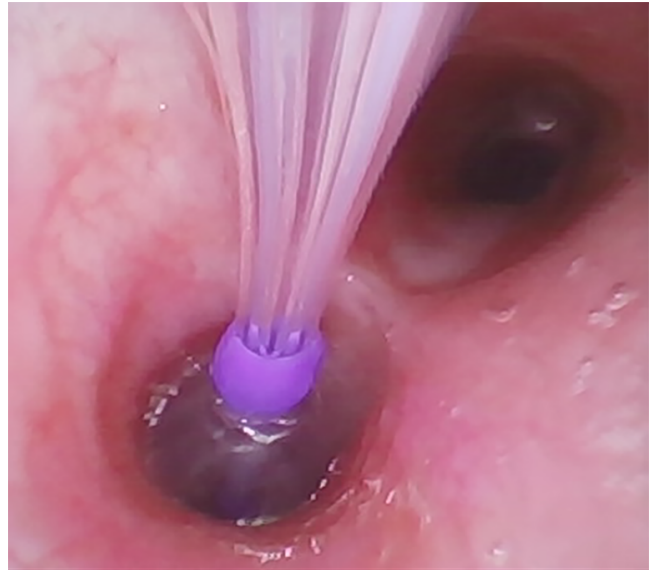

Figure 4 Process to locate the bronchial blocker (bronchoscopy group).

moderate (partial lung collapse that requires suction or manual collapse), and poor (no collapse of the lung) (12); (V) airway mucosal injury graded using BRO after surgery by an anesthetist as none (no mucosal edema), mild (mild mucosal edema), moderate (obvious mucosal edema and hyperemia), or severe (mucosal erosion and hemorrhage) (11); (VI) pulmonary infection occurred within $72 \mathrm{~h}$ after surgery, which was defined as plaque-like shadow on both lungs, with or without pleural effusion, as observed by chest X-ray; (VII) hoarseness after tracheal extubation; (VIII) duration of postoperative mechanical ventilation; (IX) duration of postoperative ICU stay; and (X) duration of postoperative hospitalization.

\section{Estimation of sample size}

The sample size was estimated with $\alpha=0.05$ and $1-\beta=0.8$ using PASS 15.0 software (NCSS, Kaysville, UT, USA). According to our previous clinical experience, the difference between groups was moderate (effect value $\mathrm{w}=0.3$ ). To determine if there were significant differences between groups according to the calculation results, each group required at least 61 patients $(\mathrm{n}=122)$.

\section{Statistical analyses}

SPSS version 15.0 software (NCSS, USA) was used for the statistical analyses. The test level was set at $\alpha=0.05$ and power was set at 0.8 . Demographic data between the 1 groups was analyzed using independent $t$-test and $\chi^{2}$ test, including age, sex, weight, and height. ASA class and thoracic surgery type between groups were analyzed using $\chi^{2}$-test. The assessment of blocker operating duration between the groups was evaluated by $\chi^{2}$-test and included required time for successful blocker placement, repositioning, and the success rate of the first blocker positioning. The effectiveness and prognosis between groups was analyzed using $\chi^{2}$-test and included degree of lung collapse, grade of airway mucosal injury, grade of airway mucosal injury, pulmonary infection, and hoarseness after tracheal extubation. The duration of postoperative mechanical ventilation, ICU stay, and hospitalization was analyzed using independent $t$-test. The distribution of data was analyzed using Kolmogorov-Smirnov test. If the data were not normally distributed $(\mathrm{P}<0.1)$, Pearson's $\chi^{2}$-test with correction or Wilcoxon rank sum test were used. The level of significant differences was set at 0.05 .

\section{Results}

A total of 127 pediatric patients were assessed for eligibility. Five patients were excluded due to abnormal position of the right upper lung lobe and a further 3 patients were excluded after grouping because OLV technique was not deemed necessary by the surgeon. Finally, 119 patients were enrolled in the study (Figure 5). The 2 groups had no significant differences in terms of demographic characteristics, including age, sex, weight, height, ASA class, and thoracic surgery type $(\mathrm{P}>0.05)$ (Table 1$)$.

The required time for successful blocker placement was significantly longer in the CT group than in the BRO group $(124.9 \pm 34.2$ vs. $92.9 \pm 17.6 \mathrm{~s}, \mathrm{P}<0.001)$. Successful blocker placement required more repositionings in the CT group than in the BRO group \{median (range), 1 [1-4] vs. $1[1-3]\}$. The success rate of first blocker positioning was significantly lower in the CT group than in the BRO group $(82.8 \%$ vs. $96.7 \%, \mathrm{P}<0.05)$ (Table 2). The degree of lung collapse was excellent in $56(96.6 \%)$ versus $61(100 \%)$ patients and fair in 2 versus 0 in the BRO and CT groups, respectively $(\mathrm{P}=0.235)$. The grade of airway mucosal injury was none in $56(96.6 \%)$ versus $60(98.3 \%)$ patients and mild in 1 versus 2 in the BRO and CT groups, respectively $(\mathrm{P}=0.965)$. Pulmonary infection within $72 \mathrm{~h}$ after surgery was observed in $56(97 \%)$ versus $59(97 \%)$ patients, and was not present in 2 versus 2 patients in the BRO and CT groups, respectively $(\mathrm{P}=1.000)$. Hoarseness after tracheal extubation also was not observed in $58(95 \%)$ versus 55 (95\%) patients in the BRO and CT groups, respectively $(\mathrm{P}=1.000)$. In addition, there was no statistically significant 


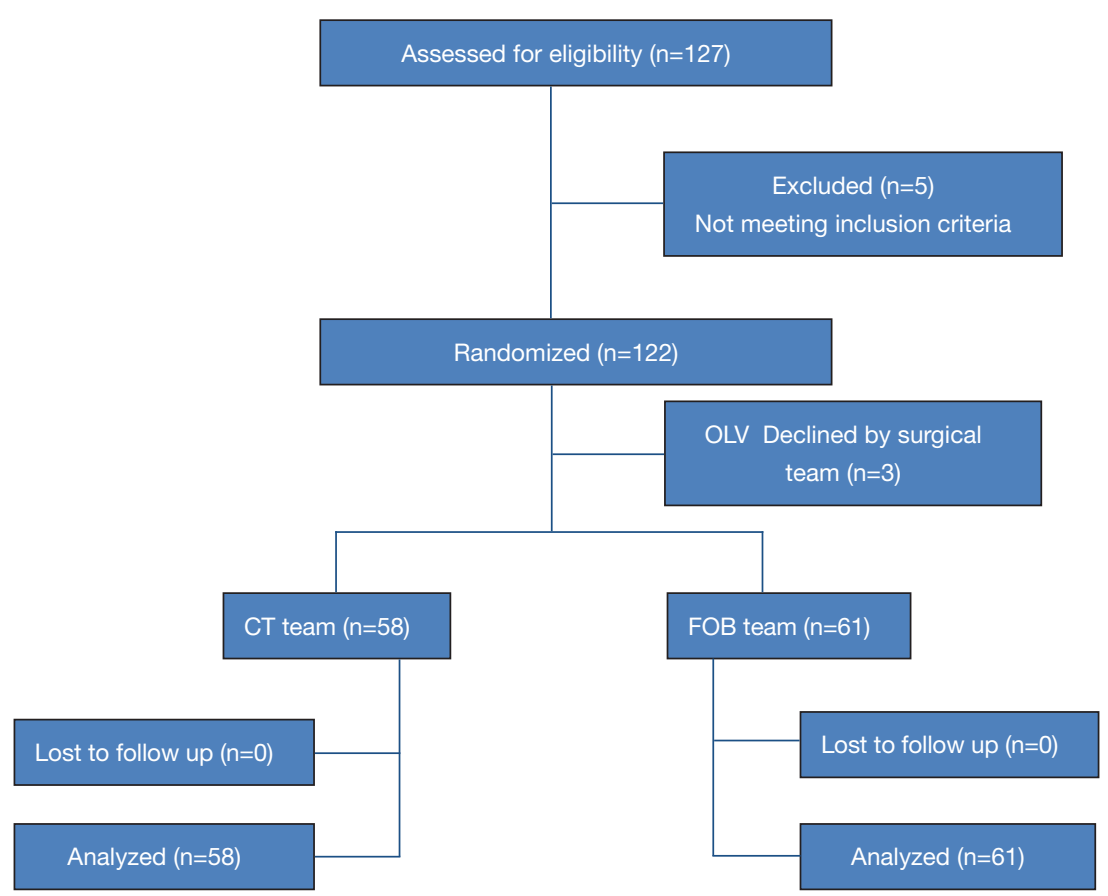

Figure 5 Consort flow diagram of the recruitment of 127 patients used in the analysis. CT, computed tomography; OLV, one-lung ventilation.

difference between the BRO and CT groups regarding the duration of postoperative mechanical ventilation $(29.1 \pm 62.7$ vs. $34.3 \pm 95.6, \mathrm{P}=0.726)$, ICU stay $(2.5 \pm 3.2$ vs. $2.9 \pm 6.3$, $\mathrm{P}=0.658)$, and hospitalization $(13.3 \pm 7.6$ vs. $13.6 \pm 11.2$, $\mathrm{P}=0.882$ ).

\section{Discussion}

The insertion of the finest $5 \mathrm{~F}$ endobronchial blocker requires a tracheal tube with an internal diameter $>4.5 \mathrm{~mm}$ (13). When applying OLV in pediatric patients, BRO cannot be used to guide intraluminal blocker placement if the insertion of a tracheal tube with an internal diameter $>4.5 \mathrm{~mm}$ is not applicable. In the present study, we used chest 3D CT evaluation to measure the airway and guide endobronchial blocker placement. CT-guided endobronchial placement is as effective with similar side effects as direct visualization via BRO for endobronchial blocker placement. This technique may be used for patients in patients in whom intraluminal BRO for endobronchial blocker placement is impossible, such as neonates and premature infants.

While comparing the techniques used for lung isolation, safety and efficiency should be considered. The adequacy of lung collapse affects surgical exposure and is a criterion for the assessment of successful OLV (14). In the present study, CT-guided endobronchial blocker placement achieved similar adequacy of lung collapse compared with BRO-guided blocker placement in pediatric patients. The balloons of most endobronchial blockers have a small volume and high pressure, and excessive inflation may induce pressure mucosal injury. During balloon-inflation procedures, the advantage of direct vision under BRO is considered to be important for the prevention of mucosal injury in small bronchi caused by balloon inflation (15). However, in the present study, no significant difference in airway mucosal injury was observed between the BRO and CT groups, suggesting that CT-guided endobronchial blocker placement would not increase the risk of airway mucosal injury. In addition, the rates of postoperative pulmonary infection and hoarseness were similar in the 2 groups $(\mathrm{P}>0.05)$. Regarding postoperative pulmonary recovery, no significant differences in the durations of mechanical ventilation, ICU stay, and hospitalization were observed between the 2 groups. Therefore, we consider that using 3D CT airway evaluation to guide endobronchial blocker placement is applicable and safe.

We postulated that 3D CT evaluation may be used to accurately estimate the insertion depth of the tracheal tube 
Table 1 Demographic characteristics, analgesia, and surgery type in the 2 groups of patients

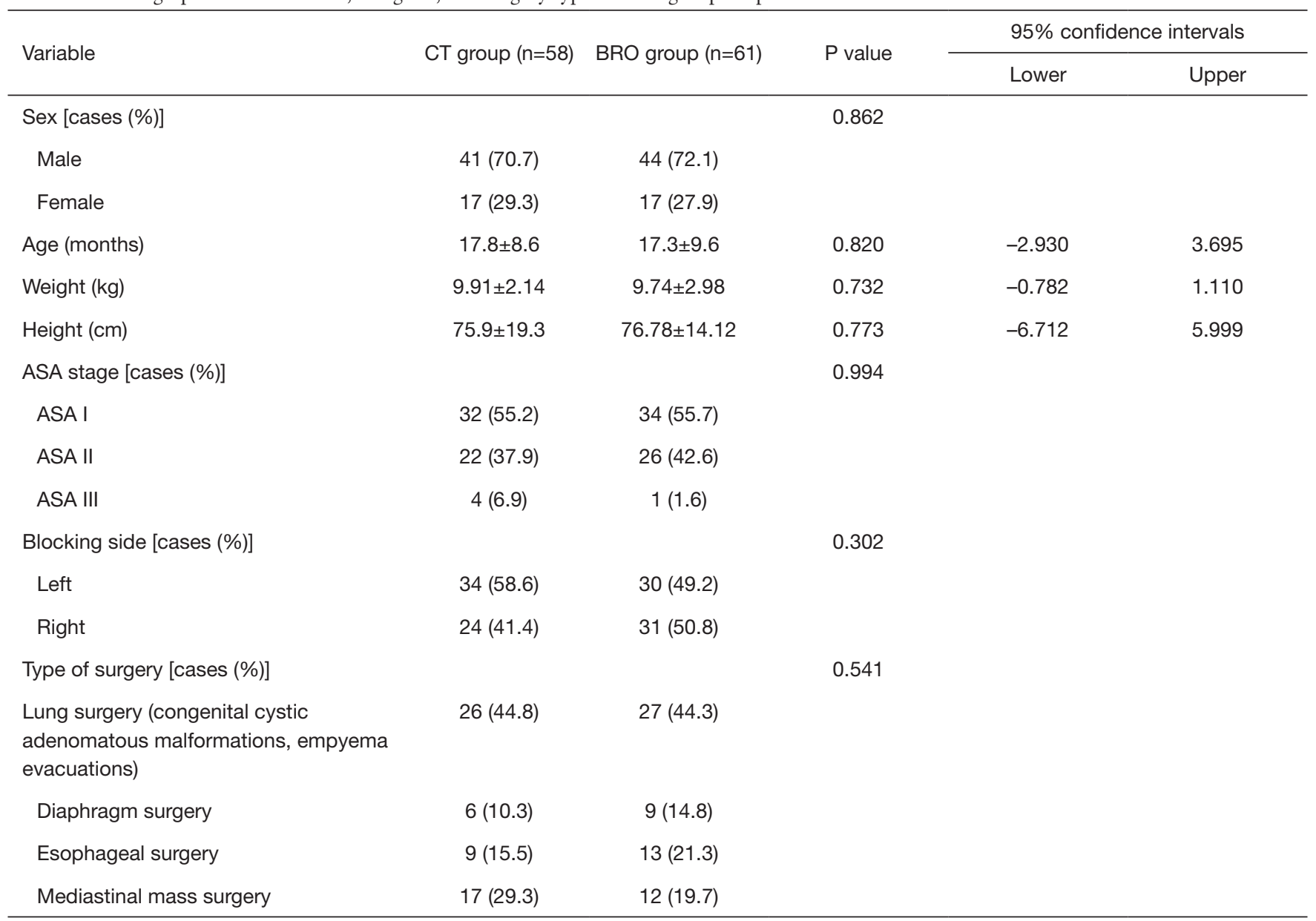

ASA, American society of Aneshesiologists; CT, computed tomography; BRO, bronchoscopy.

before blocking. The distance from point $\mathrm{A}$ to point $\mathrm{B}$ on the tip of the endobronchial blocker is approximately $2 \mathrm{~cm}$. Inserting the uncuffed tracheal tube without side holes to $2 \mathrm{~cm}$ above the carina would leave enough space to allow the insertion of the endobronchial blocker for bronchus blocking (2). In addition, by checking the markers on the endobronchial blocker during insertion, we could determine the optimal insertion depth in the bronchus of the blocking side. Therefore, 3D CT evaluation-guided endobronchial blocker placement can be used for quick intraluminal blocking.

Narayanaswamy et al. reported that the median time to complete the placement procedures under the guidance of fiberoptic BRO was $203 \mathrm{~s}$ (16), whereas Campos et al. reported a duration of $158 \mathrm{~s}$ (12). Liang et al. reported that the placement time was $185 \mathrm{~s}$ (17). In the present study, the median time to complete the placement procedures was
$92.9 \mathrm{~s}$ in the BRO group and $124.9 \mathrm{~s}$ in the CT group; both were much shorter than those reported in the literature. The reason for this could be that endobronchial blocking was performed by anesthetists with 6 years' experience of thoracic anesthesia in our center, who were skilled in performing both endobronchial blocking and BRO (18). In the present study, the required time was longer in the CT group than in the BRO group because CT-guided blocker placement required the assistance of auscultation, which required longer time than placing the blocker under direct vision through BRO.

The present study demonstrated that the number of repositionings for successful blocker placement was greater in the CT group than in the BRO group, with a lower success rate of first blocker positioning in the CT group. In some cases, it is more difficult to place bronchial blockers under CT guidance. For example, if the bronchus were 
Table 2 Comparison of endobronchial blocker placement and postoperative recovery between the 2 groups

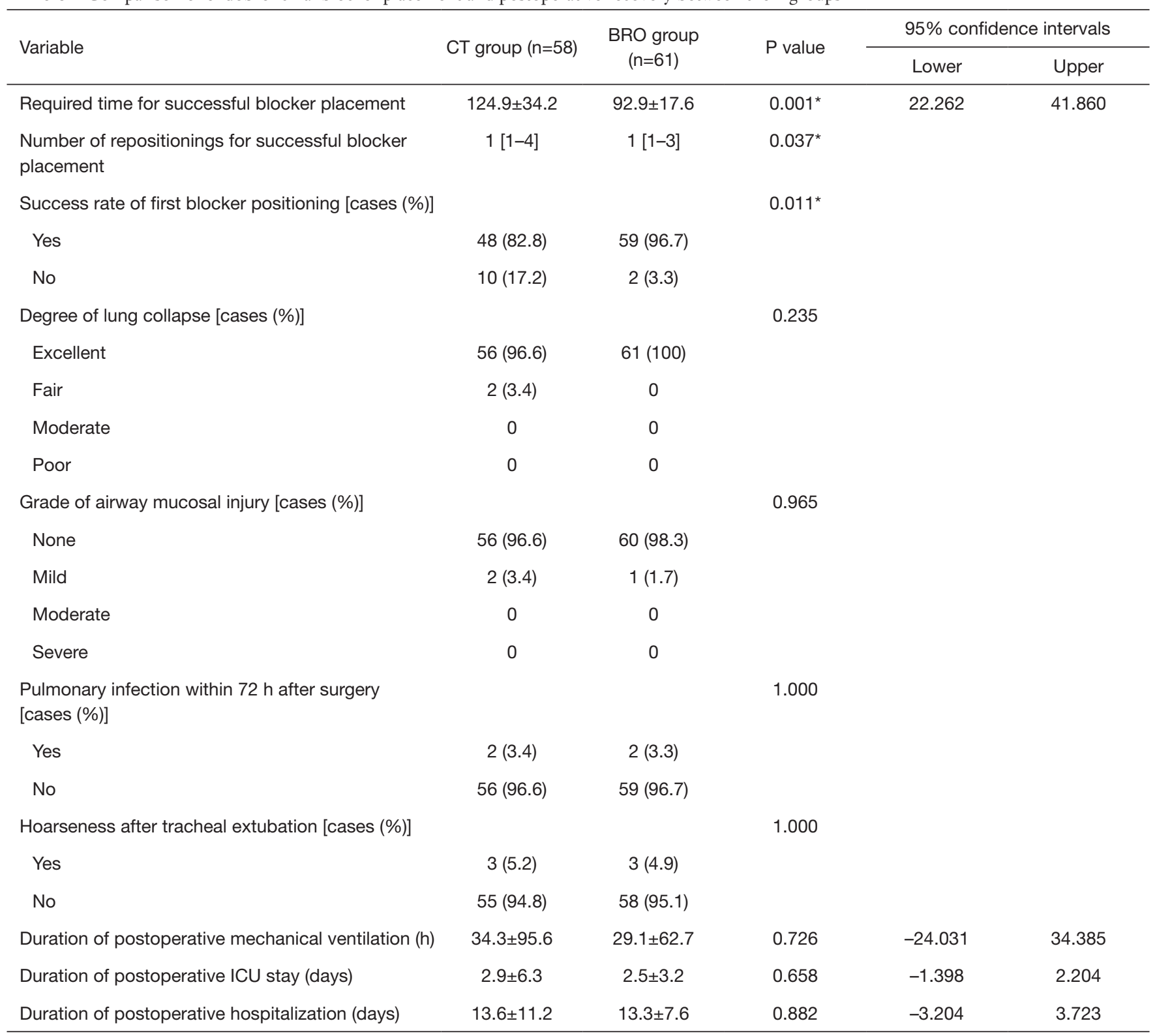

${ }^{*} \mathrm{P}<0.05$ indicates a significant difference between the 2 groups. CT, computed tomography; BRO, bronchoscopy; ICU, intensive care unit.

pulled because of pulmonary lesions, the angle between the obstructed bronchus and the coronal section is larger, or the angle between the main bronchus and the trachea is larger. When direct-vision BRO is not applicable, the following measures could be taken: (I) fixing the tracheal tube to the mouth corner at the non-blocking side, making the tube outlet face the bronchus at the blocking side, and inserting the sterile guidewire through the exhaust hole on the blocker (Figure 4A); and (II) shifting patients to the lateral position on the non-blocking side. In addition, considering that CT could be used to measure the above-mentioned angles, this problem may be solved by gradually increasing the angle contained by the tracheal tube and the blocker to the CT estimate during the blocker insertion.

The present study has some limitations. First, the major limitation was that the adequacy of lung collapse relied on surgeons' subjective assessment. Second, this was a singlecenter study, and the feasibility of using 3D CT airway evaluation to guide endobronchial blocker placement needs to be validated in future multi-center studies. Third, 
pediatric patients with abnormal position of the right upper lung lobe were excluded. The application of CT-guided endobronchial blocker placement in these patients needs further investigation.

In conclusion, compared with BRO-guided endobronchial blocker placement, CT-guided blocker placement achieved similar adequacy of lung collapse. Although CT guidance may increase the required time and repositioning for successful blocker placement, it will not increase bronchus mucosal injury and will not affect postoperative pulmonary recovery. Therefore, for pediatric patients who need to undergo surgery with OLV, 3D CT airway evaluation is a simple and efficient technique for endobronchial blocker placement.

\section{Acknowledgments}

Funding: None.

\section{Footnote}

Reporting Checklist: The authors have completed the STROBE reporting checklist. Available at http://dx.doi. org/10.21037/tp-21-33

Data Sharing Statement: Available at http://dx.doi. org/10.21037/tp-21-33

Conflicts of Interest: All authors have completed the ICMJE uniform disclosure form (available at http://dx.doi. org/10.21037/tp-21-33). The authors have no conflicts of interest to declare.

Ethical Statement: The authors are accountable for all aspects of the work in ensuring that questions related to the accuracy or integrity of any part of the work are appropriately investigated and resolved. The present study was approved by the institutional ethical committee of Guangzhou Women and Children's Medical Center (No. 2014051229). All procedures involving human participants were done in accordance with the Helsinki Declaration (as revised in 2013). Informed consent was obtained from all individual participant's parents/guardians included in the study. Images relating to participants in the manuscript were obtained with written informed consent from the parents/ guardians.
Open Access Statement: This is an Open Access article distributed in accordance with the Creative Commons Attribution-NonCommercial-NoDerivs 4.0 International License (CC BY-NC-ND 4.0), which permits the noncommercial replication and distribution of the article with the strict proviso that no changes or edits are made and the original work is properly cited (including links to both the formal publication through the relevant DOI and the license). See: https://creativecommons.org/licenses/by-nc-nd/4.0/.

\section{References}

1. Yamashita A, Okamoto H. Anesthesia for Thoracoscopic Surgery in Children. Masui 2016;65:930-6.

2. Disma N, Mameli L, Pini-Prato A, et al. One lung ventilation with Arndt pediatric bronchial blocker for thoracoscopic surgery in children: a unicentric experience. Paediatr Anaesth 2011;21:465-7.

3. Templeton TW, Lawrence AE, Lee AJ, et al. Inside out: Repurposing endobronchial intubation to facilitate extraluminal placement of a $5 \mathrm{Fr}$ Arndt bronchial blocker in young infants. Paediatr Anaesth 2018;28:668-9.

4. Hammer GB. Pediatric thoracic anesthesia. Anesth Analg 2001;92:1449-64.

5. Pawar DK, Marraro GA. One lung ventilation in infants and children: experience with Marraro double lumen tube. Paediatr Anaesth 2005;15:204-8.

6. Templeton TW, Downard MG, Simpson CR, et al. Bending the rules: a novel approach to placement and retrospective experience with the 5 French Arndt endobronchial blocker in children $<2$ years. Paediatr Anaesth 2016;26:512-20.

7. Templeton TW, Morris BN, Goenaga-Diaz EJ, et al. A Prospective Comparison of Intraluminal and Extraluminal Placement of the 9-French Arndt Bronchial Blocker in Adult Thoracic Surgery Patients. J Cardiothorac Vasc Anesth 2017;31:1335-40.

8. Wald SH, Mahajan A, Kaplan MB, et al. Experience with the Arndt paediatric bronchial blocker. Br J Anaesth 2005;94:92-4.

9. Liu Z, Zhao L, Jia Q, et al. Chest Computed Tomography Image for Accurately Predicting the Optimal Insertion Depth of Left-Sided Double-Lumen Tube. J Cardiothorac Vasc Anesth 2018;32:855-9.

10. Liu Z, Zhao L, Zhu Y, et al. The efficacy and adverse effects of the Uniblocker and left-side double-lumen tube 
for one-lung ventilation under the guidance of chest CT.

Exp Ther Med 2020;19:2751-6.

11. Forrest P. Advanced Paediatric Life Support: The Practical Approach. Anaesthesia \& Intensive Care 2001;29:440.

12. Campos JH, Kernstine KH. A comparison of a left-sided Broncho-Cath with the torque control blocker univent and the wire-guided blocker. Anesth Analg 2003;96:283-9, table of contents

13. Mohtar S, Hui TWC, Irwin MG. Anesthetic management of thoracoscopic resection of lung lesions in small children. Paediatr Anaesth 2018;28:1035-42.

14. Lu Y, Dai W, Zong Z, et al. Bronchial Blocker Versus Left Double-Lumen Endotracheal Tube for One-Lung Ventilation in Right Video-Assisted Thoracoscopic Surgery. J Cardiothorac Vasc Anesth 2018;32:297-301.

15. Borchardt RA, LaQuaglia MP, McDowall RH, et al. Bronchial injury during lung isolation in a pediatric patient. Anesth Analg 1998;87:324-5.

Cite this article as: Xu Y, Li L, Hou J, Zhang N, Zeng M, Qiu Q, Liang Y, Wei W, Tan Y. 3D CT airway evaluationguided intraluminal placement of endobronchial blocker in pediatric patients: a randomized controlled study. Transl Pediatr 2021;10(3):625-634. doi: 10.21037/tp-21-33
16. Narayanaswamy $M, M c R a e ~ K$, Slinger $P$, et al. Choosing a lung isolation device for thoracic surgery: a randomized trial of three bronchial blockers versus double-lumen tubes. Anesth Analg 2009;108:1097-101.

17. Liang P, Ni J, Zhou C, et al. Efficacy of a New Blind Insertion Technique of Arndt Endobronchial Blocker for Lung Isolation: Comparison With Conventional Bronchoscope-Guided Insertion Technique-A Pilot Study. Medicine (Baltimore) 2016;95:e3687.

18. Campos JH, Hallam EA, Van Natta T, et al. Devices for lung isolation used by anesthesiologists with limited thoracic experience: comparison of double-lumen endotracheal tube, Univent torque control blocker, and Arndt wire-guided endobronchial blocker. Anesthesiology 2006;104:261-6, discussion 5A.

(English Language Editor: R. Scott) 